\title{
GIST or Not - A Unique Case of Low Grade Endometrial Stromal Sarcoma with Review of Literature
}

\section{Sreekala Sreehari* and Balaji Balasubramanian}

NMC Specialty Hospital, Abudhabi, United Arab Emirates

*Corresponding author: Sreehari S, NMC Specialty Hospital, Abudhabi, United Arab Emirates, Tel: +971 4 267 9999; E-mail: sreekalasreehari@gmail.com Received date: July 08, 2015; Accepted date: April 15, 2016; Published date: May 05, 2016

Copyright: ( 2016 Sreehari S, et al. This is an open-access article distributed under the terms of the Creative Commons Attribution License, which permits unrestricted use, distribution, and reproduction in any medium, provided the original author and source are credited.

\begin{abstract}
Endometrial stromal sarcoma (ESS) is a mesenchymal neoplasm that usually occurs as a primary tumor of the uterine corpus, but rarely arises in other sites, such as the ovary, pelvic cavity, mesentery, omentum and intestine. Endometrial stromal sarcoma is a malignant subtype of endometrial stromal tumor arising from the stroma (connective tissue) of the endometrium rather than the glands. There are three grades for endometrial stromal tumors. All the cases yet reported in literature had a history of previous hysterectomy with or without a diagnosis of ESS. Here we present a unique case of Low grade ESS where the patient presented with vague abdominal pain, loss of weight and anemia. On evaluation with USG and CT demonstrated a lobulated mass in mesentery which led to the diagnosis of a GIST from small bowel. Intraoperatively the lesion was in omentum with no attachment to mesentery. The enlarged uterus with fibroids were also removed along with the omental mass after a clinicopathological discussion which suggested the possibility of ESS with primary in the uterus. The histopathology demonstrated Low grade Endometrial sarcoma which was supported by immunohistochemistry.
\end{abstract}

Keywords: Endometrial stromal sarcoma; Neoplasm

\section{Background}

- Unusual lesion: endometrial stromal sarcoma

- Unusual presentation: masquerading as GIST and intraoperatively as an omental mass with no relation to uterus!

- The nodule in the uterus is totally hyalinised.

- Immunophenotype and PR positivity with treatment impact

\section{Case Presentation}

A 45 year old woman, gravida 2, para 2, presented with vague abdominal pain for 3-4 months. She had noticed recent weight loss of about $5 \mathrm{~kg}$ in 4 months and loss of appetite. She had dyspnea on exertion since 3 months. She has no comorbidity. She is not a smoker or alcoholic. In the past, Tubal ligation was done after 2 nd delivery. She has no family history of cancers.

\section{Investigations}

On evaluation her general condition was unremarkable. Per abdomen examination showed a large firm mass in the supraumbilical region which is mobile in transverse axis. No gross supraclavicular nodes were felt. Her investigations were reviewed. Routine blood tests, blood chemistry and urine test revealed no abnormal findings, apart from a Haemoglobin value of $9.2 \mathrm{gm} \%$. USG abdomen revealed a large hypoechoic lesion in the central abdomen with lobulated margins measuring $8.7 \times 6.6 \mathrm{~cm}$. Uterus was bulky with multiple fibroids.

CT scan of the abdomen was done with contrast to further delineate the lesion. This showed $10 \mathrm{~cm}$ lobulated lesion in the abdomen possibly in the mesentery and splaying the small bowel. Clinicoradiologically, possibility of GIST from the small bowel was thought (Figure 1).

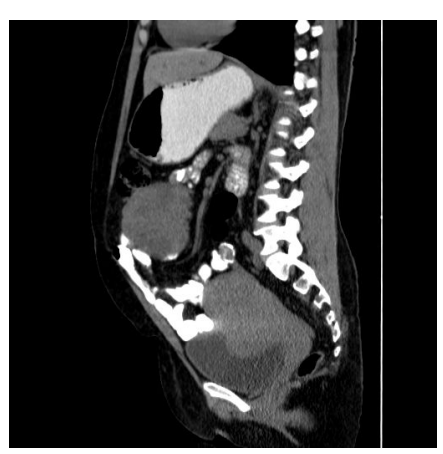

Figure 1: CT scan of the abdomen.

\section{Treatment}

The case was discussed in the tumor board and the surgery was planned for explorative laparotomy and resection of the tumor. During Laparotomy, the tumor was found to be in omentum with no relation to the bowel or mesentery (Figure 2). Intraoperative clinicopathological discussion was done. Although a primary omental GIST was still a possibility, the bulky uterus with multiple fibroids, 2 of them which were exophytic yellowish and adherent to the omentum gave the possibility of a primary mesenchymal tumour with omental metastasis. Since all other organs were normal and no other primary lesion could be made out, total abdominal hysterectomy and omentectomy was done. 


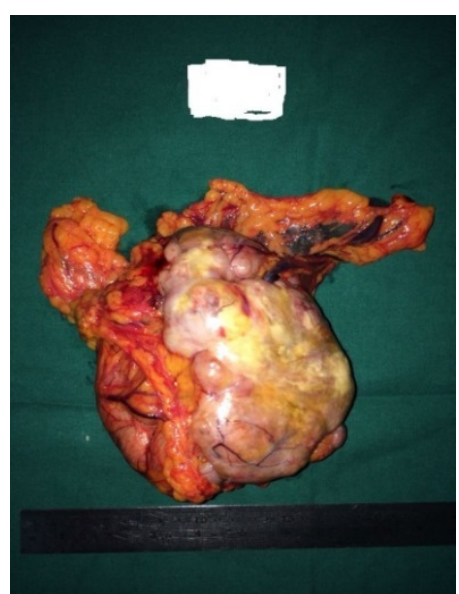

Figure 2: Gross picture of the tumour.

Intraoperative clinicopathological discussion was done. Although a primary omental GIST was still a possibility, the bulky uterus with multiple fibroids, 2 of them which were exophytic yellowish and adherent to the omentum gave the possibility of a primary mesenchymal tumour with omental metastasis. Since all other organs were normal and no other primary lesion could be made out, total abdominal hysterectomy and omentectomy was done.

Grossly, four specimens were received; one omental mass, 2 separate fibroids and uterus with cervix including both tubal stumps and ovaries. The omental mass (Figure 2) was a well encapsulated nodular grey white to yellowish solid fleshy lesion measuring $8 \times 5 \times 3 \mathrm{~cm}$, lesion with areas of necrosis. Microscopically, the lesion showed sheets of oval to spindly plump cells resembling endometrial stroma (Figure $3)$.

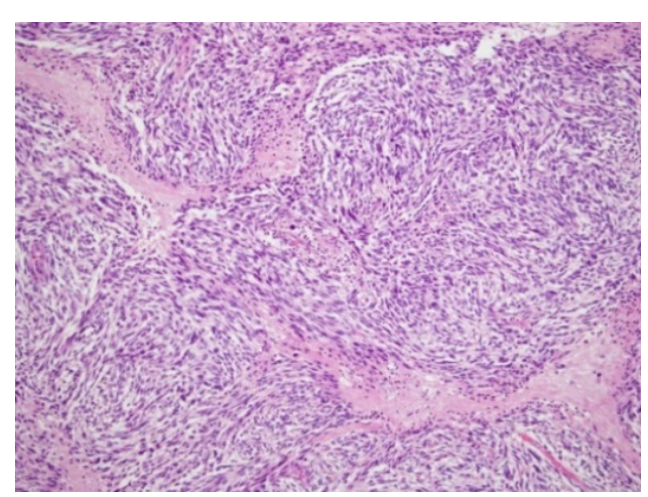

Figure 3: Microscopy of endometrial stroma.

The cells had small amount of cytoplasm and dense chromatin. Mitosis upto 30-35/ 10 hpf seen. Apoptotic debris and areas of necrosis noted. A rich network of capillaries with a conspicous perivascular pattern was noted. Immunohistochemically, the tumour cells were positive for $\mathrm{CD} 10$ and $\mathrm{Bcl} 2$. The Progesterone receptor showed positivity. The cells were negative for SMA, Desmin, CD34 and ER.

The uterus showed multiple intramural subserous and submucous fibroids with no evidence of similar lesion as in the omentum. Both the ovaries and tubal stumps were negative. One of the separately received fibroids show extensive hyalinization with calcification and foamy histiocytic infiltration which totally replaced the primary disease process. The marked area of fundus of uterus from where this fibroid was taken was extensively sampled. But no evidence of ESS could be found.

\section{Outcome and follow-up}

Finally, the diagnosis was given as Endometrial Stromal sarcoma, low grade presenting as omental mass and a comment added that the hyalinised lesion might have been a primary which regressed. Her postoperative recovery was uneventful. A follow up PET scan was done which revealed no other lesion. She was started on Medroxy progesterone since then. She is on follow up and free of disease since 6 months.

\section{Discussion}

Endometrial stromal tumours (ESTs) of the uterus are the second most common mesenchymal tumours of the uterus eventhough they account for $10 \%$ of all such tumours [1]. In the 2003 World Health Organization classification [2] ESTs are divided into:

- Endometrial Stromal Nodule (ESN),

- Low-grade Endometrial Stromal Sarcoma (ESS),

- Undifferentiated Endometrial Sarcoma (UES).

The ESN and the low-grade ESS fall in the lower end of the spectrum of this group of tumors. Both are typically composed of a diffuse growth of small blue cells with scant cytoplasm, and oval to spindle nuclei that resemble the endometrial stromal cells of the proliferative endometrium [1,2]. Myometrial invasion and lymphovascular emboli are the criteria to distinguish between these.

The main diagnostic criteria to distinguish low-grade and undifferentiated endometrial sarcoma is based on histomorphological features as defined by WHO undifferentiated endometrial sarcomas display marked cellular atypia and lack morphologic evidence of an endometrial stromal phenotype' along with immunophenotype of ESS.

In distinguishing endometrial stromal sarcoma from uterine smooth muscle neoplasms, $\mathrm{CD} 10$ and $\mathrm{H}$-caldesmon has been touted as more specific markers whereas desmin, smooth muscle actin, musclespecific actin and calponin have all been variably shown to stain endometrial sarcomas.

Low-grade endometrial stromal sarcoma was more often positive for ER and PR [3] whereas a higher percentage of undifferentiated endometrial sarcomas are negative.

The fusion of the two zinc-finger genes JAZF1 and SUZ1 at the 7p15 and $17 \mathrm{q} 21$ breakpoints respectively was described in endometrial stromal tumors by Koontz et al. in 2001 [4]. The function of this fusion transcript is still unknown. The prevalence of the associated translocation, $t(7 ; 17)$ (p15; $\mathrm{q} 21)$ in lowgrade endometrial stromal sarcoma by RTPCR 10, 13, 14, 16 and FISH11, 12 ranges from 23 to $80 \%$. Other rearrangements include with the PHF1 (6p21) zinc-finger gene have been described in $1 \%$ of low-grade endometrial stromal sarcomas.

Chang et al. [5] reported that neither mitotic index nor cytologic atypia in primary extrauterine ESS were predictive of tumor recurrence or death from tumor. In their series, $77 \%$ of patients whose tumors had 
Page 3 of 3

a mitotic index of less than 10 per HPF had one or more recurrences and $30 \%$ died from their neoplasm. The behavior of the primary extrauterine ESS was more reminiscent of high-grade primary uterine ESS than low-grade primary uterine ESS. Therefore, close observation and extended follow-up will be required in extrauterine ESS, regardless of mitotic activity. By far the most reliable prognostic factor in uterine sarcomas is stage. Literature review has shown that the majority of uterine sarcomas in general present at stage I, i.e. limited to the uterus. For this reason primary extrauterine endometrial sarcomas, which presents outside the uterus has to be staged and managed as a higher stage sarcoma.

In general, extrauterine endometrial stromal sarcomas are quite rare. Endometrial stromal sarcoma arising from endometriosis is well reported in literature [6-8]. Majority of about $75 \%$ are evident in ovary while $25 \%$ involve other organs.

In our case, uterus had only multiple fibroids with no omental adhesions. The omentum was only site of the tumor. Sections from omentum, uterus and adnexae didn't reveal any foci of endometriosis. Oestrogen and progesterone are important regulators of endometrial stromal function and act by binding to their nuclear receptors. In analogy to breast carcinoma, there is heterogeneous expression of ER and PR in LGSSs on a cellular level, which suggests different levels of susceptibility to hormonal therapy [3].

In our case the presence of hyalinised subserous nodule presented initially with the confusion whether to hypothesise as a primary site which underwent hyalinisation. But extensive sampling didn't reveal any reliable evidence for the same. The presentation as a solitary omental mass with presence of lymphovascular invasion, absence of any other lesion on PET scan and positivity for PR are the determining factors for follow up and further management in our case. Because of the rarity of such cases, very little is known about the exact pathogenesis.

Since the disease has known to recur after a long latent period [2,9] long term follow up is advised in these cases. Adjuvant therapy with progestin is reasonable due to the hormone receptor status.

The enlarged uterus with fibroids were also removed along with the omental mass after a clinicopathological discussion which suggested the possibility of ESS with primary in the uterus. The histopathology demonstrated Low grade Endometrial sarcoma which was supported by immunohistochemistry.

\section{Learning Points/Take Home Messages}

- Endometrial stromal sarcoma needs to be considered in the differential diagnosis of a female with abdominal mass, even without a prior history.

- Intraoperative clinicopathological discussions can shed more lights on diagnosis and can alter the surgical procedures avoiding repeat surgeries.

- Immunohistochemitry is a great aid in diagnosing and prognosticating Endometrial stromal sarcoma with hormonal receptors useful in further management.

\section{References}

1. Baker P, Oliva E (2007) Endometrial stromal tumours of the uterus: a practical approach using conventional morphology and ancillary techniques. J Clin Pathol 60: 235-243.

2. Hendrickson MR, Tavassoli FA, Kempson RL (2003) Mesenchymal tumors and related lesions. Pathology and genetics of tumours of the breast and female organs. Lyon: IARC Press pp: 233-236.

3. Reich O, Regauer S, Urdl W, Lahousen M, Winter R (2000) Expression of oestrogen and progesterone receptors in low-grade endometrial stromal sarcomas. British Journal of Cancer 82: 1030-1034.

4. Jakate K1, Azimi F, Ali RH, Lee CH, Clarke BA, et al. (2013) Endometrial sarcomas: an immunohistochemical and JAZF1 re-arrangement study in low-grade and undifferentiated tumors. Modern Pathology 26: 95-105.

5. Chang KL, Crabtree GS, Lim-Tan SK, Kempson RL, Hendrickson MR (1993) Primary extrauterine endometrial stromal neoplasms: a clinicopathologic study of 20 cases and a review of the literature. Int J Gynecol Pathol 12: 282-96.

6. Kovac D, Gasparović I, Jasic M, Fuckar D, Dobi-Babić R (2010) Endometrial stromal sarcoma arising in extrauterine endometriosis: a case report. J Minim Invasive Gynecol 17: 541-542.

7. Sinha R, Sundaram M (2005) Endometrial stromal sarcoma from endometriosis. Eur J Gynaecol Oncol. 26: 113-6.

8. Lan C, Huang X, Lin S, Cai M, Liu J (2012) Endometrial stromal sarcoma arising from endometriosis: a clinicopathological study and literature review. Gynecol Obstet Invest 74: 288-297.

9. Ashraf-Ganjoei T, Behtash N, Shariat M, Mosavi A (2006) Low grade Endometrial Stromal Sarcoma of uterine corpus, a clinico-pathological and survey study in 14 cases. World Journal of Surgical Oncolog 4: 50. 